\title{
Pulmonary artery pressure: early predictor of chronic lung disease in preterm infants
}

\author{
N V Subhedar, A H Hamdan, S W Ryan, N J Shaw
}

\begin{abstract}
Aim-To determine if pulmonary artery pressure (PAP) in ventilated preterm infants is independently associated with the development of chronic lung disease (CLD) and whether early assessment has any prognostic value.

Methods-Two cohorts (development $\mathbf{n}=$ 55; and validation $n=28$ ) of preterm infants were studied at 24 hours of age. PAP was assessed non-invasively using its inverse correlation with the corrected acceleration time to right ventricular ejection time ratio (AT:RVET(c)), calculated from the pulmonary artery Doppler waveform. Clinical and respiratory variables were also collected. Using logistic regression analysis to identify factors independently associated with CLD, a prognostic score was developed to predict CLD. The ability of the score to predict CLD was described using receiver operating characteristic (ROC) curves.

Results-Birthweight, inspired oxygen concentration, and AT:RVET(c) were independently predictive of CLD. The area under the ROC curve was 0.96 for the development and 0.89 for the validation cohort. Exclusion of AT:RVET(c) resulted in a reduction to 0.88 and 0.73 , respectively.

Conclusion-PAP is independently associated with CLD. An early assessment of PAP using AT:RVET(c) may permit the early prediction of CLD as part of a multifactorial scoring system.
\end{abstract}

(Arch Dis Child Fetal Neonatal Ed 1998;78:F20-F24)

Keywords: pulmonary artery pressure; chronic lung disease; AT:RVET(c) ratio; inspired oxygen concentration

Department of Child Health, University of Liverpool N V Subhedar S W Ryan

Regional Neonatal Intensive Care Unit, Liverpool Women's Hospital

A H Hamdan

N J Shaw

Correspondence to: Dr N V Subhedar Regional Neonatal Intensive Care Unit,

Liverpool Women's Hospital, Liverpool L8 7SS.

Accepted 29 May 1997 using a combination of demographic, clinical, and respiratory variables, most lack the ability to predict outcome at an early stage in the disease process..$^{3-6}$ Failure of early postnatal cardiovascular adaptation, with persistently increased pulmonary artery pressure (PAP), is associated with an increased incidence of neonatal complications. ${ }^{7}$ However, it is unclear if this effect is independent of other factors which adversely affect prognosis, and in particular, whether the influence of PAP is independent of other markers of disease severity.

Assessment of PAP may be useful in predicting adverse respiratory outcome in preterm infants. However, as a single indicator it lacks the necessary specificity required to be an early prognostic marker. ${ }^{8}$ Doppler echocardiography allows PAP to be estimated non-invasively using systolic time intervals derived from the pulmonary artery velocity waveform. The corrected acceleration time to right ventricular ejection time ratio (AT:RVET(c)) is inversely related to PAP measured directly and has been widely used to follow pulmonary haemodynamics in preterm infants. ${ }^{9-11}$

\section{Methods}

Preterm infants of less than 32 weeks of gestation admitted to the neonatal intensive care unit were studied between 1994 and 1996. Two separate cohorts were studied: an initial development cohort in whom an assessment of PAP was made and its association with other clinical and physiological factors investigated; and a validation cohort in whom the performance of the predictive score was evaluated. All infants with early onset respiratory distress received two doses of surfactant, either natural or artificial, immediately after initial intubation and stabilisation and again at 12 hours of age. Infants were enrolled into the study if they continued to receive mechanical ventilation at 24 hours of age. Basic demographic data, details of ventilatory support, and arterial blood gas results were recorded at entry into the study. Parental consent was obtained for each infant studied and the study was approved by the local paediatric ethics committee.

Doppler echocardiography was performed using either an ATL Ultramark IV ultrasound scanner (development cohort) or a Diasonics Vingmed CFM 725 system (validation cohort). A 7.5 Mhz multifrequency imaging transducer combined with a $5 \mathrm{MHz}$ Doppler transducer was used in each case. Doppler ultrasound examination was performed as described before. ${ }^{12}$ In brief, a two dimensional image of the main pulmonary artery was obtained using a parasternal short axis view and a Doppler 
signal was recorded from the centre of the artery, immediately distal to the valve leaflets. Measurements of the acceleration time and right ventricular ejection time were made from the Doppler velocity waveform. The AT:RVET(c) was calculated by dividing the ratio between the acceleration time and right ventricular ejection time by the square root of the R-R interval from a simultaneous ECG tracing. The mean AT:RVET(c) ratio from three to five waveforms was used for subsequent data analysis. The intraobserver reproducibility of this technique has been reported recently. ${ }^{12}$

In infants in the validation cohort the presence of a patent ductus arteriosus (PDA) and the direction of ductal shunting was assessed using a high parasternal approach with the aid of colour flow mapping. Left to right ductal flow with a left atrial to aortic root ratio greater than 1.5 was considered to represent significant ductal shunting. ${ }^{13}$ Staff involved in the daily management of infants were unaware of the results of the echocardiographic assessment, unless clinically important ductal shunting was suspected. The ultrasound system available during assessment of infants in the development cohort did not have colour Doppler capability, and therefore precluded the reliable echocardiographic diagnosis of PDA. In this group the diagnosis of a symptomatic PDA was made on the basis of typical clinical features. ${ }^{14}$ Infants with a symptomatic PDA (diagnosed clinically or on the basis of echocardiographic criteria) were treated with indomethacin $0.1 \mathrm{mg} / \mathrm{kg} /$ day for 6 days; surgical ligation was performed in infants who failed to respond to two courses of indomethacin. During the period of the study, it also became our policy to treat all infants below 1 $\mathrm{kg}$ with prophylactic indomethacin $(0.1 \mathrm{mg} / \mathrm{kg})$ shortly after birth and for two further doses at 12 hourly intervals.

Outcome data for CLD and neonatal death was collected prospectively. CLD was defined as continuing oxygen dependency for 28 days and beyond 36 weeks of postmenstrual age. ${ }^{15}$ Throughout the study period our policy was to administer supplemental oxygen to maintain arterial oxygen saturation above $93 \%$ (Ohmeda Biox 3700e pulse oximeter). Deaths which occurred before 36 weeks of postmenstrual age were classified in the CLD outcome group.

Statistical analysis of the development cohort was performed using multiple logistic

Table 1 Infants with and without CLD (development cohort) *

\begin{tabular}{llll}
\hline & No CLD $n=29$ & CLD $n=26$ & p Value \\
\hline Birthweight (g) & $1184(718-2256)$ & $745(580-1538)$ & $<0.0001$ \\
Gestational age (weeks) & $29(25-31)$ & $26(23-31)$ & $<0.0001$ \\
Antenatal steroids & $21(72)$ & $23(88)$ & 0.25 \\
Prophylactic indomethacin & $3(10)$ & $8(31)$ & 0.12 \\
Male sex & $15(52)$ & $14(54)$ & 1.0 \\
FIO $(\%)$ & $34(21-100)$ & $58(23-100)$ & 0.0001 \\
a:A ratio & $0.29(0.04-0.67)$ & $0.16(0.06-0.50)$ & 0.001 \\
PIP (cm H2O) & $14(10-24)$ & $16(12-29)$ & 0.12 \\
AT:RVET(c) & $0.47(0.41-0.53)$ & $0.44(0.37-0.51)$ & 0.0004 \\
pH & $7.34(7.16-7.41)$ & $7.26(7.10-7.41)$ & 0.0002 \\
$\mathrm{PO}_{2}$ (mm Hg) & $55(27-132)$ & $54(38-86)$ & 0.71 \\
$\mathrm{PCO}_{2}$ (mm Hg) & $38(28-68)$ & $45(35-63)$ & 0.0007 \\
$\mathrm{Symptomatic} \mathrm{PDA}$ & $2(7)$ & $7(27)$ & 0.10 \\
\hline
\end{tabular}

^ Results expressed as median (range) or number (\%). regression (SPSS Release 4.0, SPSS Inc., Chicago, USA) with CLD as the outcome variable, and birthweight, gestational age, peak inspiratory pressure (PIP), inspired oxygen concentration $\left(\mathrm{FIO}_{2}\right), \mathrm{pH}$, and $\mathrm{AT}: \operatorname{RVET}(\mathrm{c})$ as explanatory variables. These factors were selected because they represented the most important discriminatory variables when infants who developed CLD were compared with those who did not. Analysis of the correlations between continuous variables also guided the choice of the most suitable potential predictors in the development cohort; for example, as there was a close association between $\mathrm{pH}$ and $\mathrm{PCO}_{2}$, only $\mathrm{pH}$ was included in the regression analysis. A similar association existed between the arterial to alveolar oxygen tension (a:A) ratio and $\mathrm{FIO}_{2}$. The a:A ratio was excluded from the list of potential predictive variables because, unlike $\mathrm{FIO}_{2}$, it was also significantly correlated with birthweight and gestational age.

Multiple logistic regression was performed using a backward stepwise strategy, excluding variables from the model if $p>0.05$. A prognostic score was developed from the regression model incorporating only the resulting significant independent variables. A range of probability values between 0 and 1 was calculated using this score to predict CLD for the development cohort. A receiver operating characteristic (ROC) curve, a plot of sensitivity versus 1 minus specificity, was then constructed to demonstrate graphically the discriminatory performance of the score in predicting outcome across the spectrum of probability values. The validation cohort served to test prospectively the performance of this score. The area under the ROC curve permitted quantification of the performance in each cohort. Comparisons within and between cohorts were made using the Mann-Whitney U test or Fisher's exact test with Yates' correction. The correlation between continuous variables was expressed as Pearson's correlation coefficient.

\section{Results}

Eighty three infants were studied in total; fifty five in the development cohort and twenty eight in the validation cohort.

DEVELOPMENT PHASE

Table 1 shows a comparison of various demographic and physiological variables between infants developing CLD and those who did not develop CLD in the development cohort. Median birthweight and gestational age were significantly lower in infants who developed CLD; these infants also had higher median $\mathrm{FIO}_{2}$ requirements and $\mathrm{PCO}_{2}$ values, but lower median AT:RVET(c), a:A ratio, and $\mathrm{pH}$ values at 24 hours of age.

A weak but significant correlation was present between AT:RVET(c) and $\mathrm{FIO}_{2}$ (r $=-0.33, \mathrm{p}=0.01)$ and AT:RVET $(\mathrm{c})$ and $\mathrm{pH}(\mathrm{r}$ $=0.28 ; \mathrm{p}=0.03)$. There was no significant correlation between AT:RVET(c) and any of the other continuous variables listed in table 1 . A moderate correlation was present between $\mathrm{pH}$ and $\mathrm{FIO}_{2}(\mathrm{r}=-0.51 ; \mathrm{p}<0.001)$ and $\mathrm{a}$ 
closer correlation between $\mathrm{pH}$ and $\mathrm{PCO}_{2}(\mathrm{r}=-$ $0.77 ; \mathrm{p}<0.001)$. A similar correlation was found between $\mathrm{FIO}_{2}$ and a:A ratio $(\mathrm{r}=-0.75$; $\mathrm{p}<0.001)$.

REGRESSION ANALYSIS

Results of the multiple logistic regression analysis with significant independent variables associated with CLD (birthweight, $\mathrm{FIO}_{2}$, and $\mathrm{AT}: \operatorname{RVET}(\mathrm{c})$ ) and the logistic regression equation describing this correlation, are shown in table 2. Gestational age and $\mathrm{pH}$ were not independently predictive for the development of CLD and were therefore excluded from the final regression model.

VALIDATION PHASE

The characteristics of the validation cohort and development cohorts are shown in table 3 . Median birthweight and gestational age were similar in both groups. A greater proportion of infants in the validation cohort received prophylactic indomethacin. The severity of respiratory disease also seemed to be greater in the validation cohort, as reflected by a higher median PIP and a tendency to higher $\mathrm{FIO}_{2}$ requirements. The proportion of survivors who developed CLD was greater in the validation cohort.

There was no significant difference in median AT:RVET(c) between the two groups. The incidence of symptomatic PDA was also similar in the two groups. Ductal ligation was performed in one infant in each group. In the validation cohort at 24 hours of age, left to right ductal shunting was identified in nine $(32 \%)$ infants and right to left ductal flow in

Table 2 Logistic regression analysis

\begin{tabular}{lll}
\hline & Regression coefficient & Odds ratio $(95 \%$ CI) \\
\hline Birthweight $(\mathrm{g})$ & -0.014 & $0.99(0.98-0.997)$ \\
FIO $_{2}(\%)$ & 0.112 & $1.12(1.03-1.21)$ \\
AT:RVET $(\mathrm{c}) \star^{\star}$ & -0.477 & $0.62(0.43-0.90)$ \\
\hline *expressed as AT:RVET $(\mathrm{c}) \times 100$. &
\end{tabular}

Logistic equations $\star \star$

$$
\begin{aligned}
\mathrm{y}=29.92 & +\left(0.112 \times \mathrm{FIO}_{2}(\%)\right) \\
& -(47.7 \times \mathrm{AT}: \operatorname{RVET}(\mathrm{c})) \\
& -(0.014 \times \text { Birthweight }(\mathrm{g})) \\
\mathrm{p}=\frac{\mathrm{e}^{\mathrm{y}}}{1+\mathrm{e}^{\mathrm{y}}} &
\end{aligned}
$$

\begin{tabular}{|c|c|c|c|}
\hline & $\begin{array}{l}\text { Development cohort } \\
n=55\end{array}$ & $\begin{array}{l}\text { Validation cohort } \\
n=28\end{array}$ & $p$ \\
\hline Birthweight (g) & $920(580-2256)$ & $1044(520-1720)$ & 0.46 \\
\hline Gestational age (weeks) & $28(23-31)$ & $29(24-31)$ & 0.054 \\
\hline Antenatal steroids & $44(80)$ & $27(96)$ & 0.09 \\
\hline Prophylactic indomethacin & $11(20)$ & $13(46)$ & 0.02 \\
\hline Male sex & $29(53)$ & $17(61)$ & 0.64 \\
\hline $\mathrm{FIO}_{2}(\%)$ & $40(21-100)$ & $60(22-100)$ & 0.07 \\
\hline a:A ratio & $0.22(0.04-0.67)$ & $0.15(0.09-0.46)$ & 0.21 \\
\hline $\mathrm{PIP}\left(\mathrm{cm} \mathrm{H}_{2} \mathrm{O}\right)$ & $14(10-29)$ & $20(12-28)$ & 0.002 \\
\hline AT:RVET (c) & $0.47(0.37-0.53)$ & $0.45(0.25-0.68)$ & 0.51 \\
\hline Symptomatic PDA & $9(16)$ & $3(11)$ & 0.71 \\
\hline Ductal shunting & & $10(38) \dagger$ & \\
\hline CLD & $19(35)$ & $17(61)$ & 0.04 \\
\hline Deaths & $7(13)$ & $6(21)$ & 0.48 \\
\hline
\end{tabular}

${ }^{\star \star} \mathrm{e}$, base of natural logarithms. p, probability of developing CLD.

Table 3 Patient characteristics *

* Results expressed as median (range) or number (\%).

† Includes one infant with right to left ductal shunting.

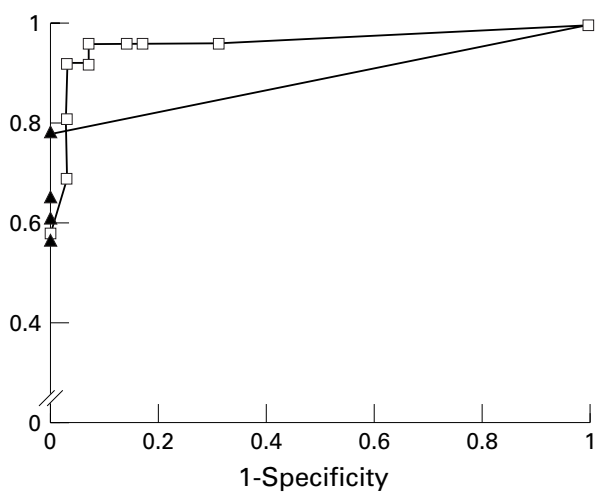

Figure 1 ROC curves for development and validation cohorts. $\square$ development; $\boldsymbol{\Delta}$ validation

Table 4 Comparison of areas under ROC curves

\begin{tabular}{ll}
\hline Cohort & $\begin{array}{l}\text { Area under } \\
\text { ROC curve }\end{array}$ \\
\hline Development & 0.96 \\
Development, excluding AT:RVET(c) & 0.88 \\
Validation & 0.89 \\
Validation, excluding AT:RVET(c) & 0.73 \\
\hline
\end{tabular}

one $(3 \%)$ infant. There was no significant difference in median AT:RVET(c) between infants with and without left to right ductal shunting ( 0.45 vs 0.44 , respectively; $\mathrm{p}=0.70)$.

PERFORMANCE OF THE SCORE

Figure 1 shows the ROC curves for the performance of the score in the development and validation cohorts. The areas under the ROC curve were 0.96 (SE 0.03) and 0.89 (SE 0.03), respectively. Exclusion of an assessment of PAP resulted in a deterioration in the performance of the score, with a reduction in the area under the ROC curve (table 4).

The precise probability cutoff value ultimately chosen would be tailored to suit requirements: for example, for a logistic probability $\mathrm{p}>0.1$, the score would yield a sensitivity of 0.78 , a specificity of 1.0 , a positive predictive value of 1.0 , and a negative predictive value of 0.5 in the validation cohort.

\section{Discussion}

This study shows that PAP is independently associated with CLD in ventilated preterm infants. We have also successfully used this relation to develop a prognostic score, which includes an assessment of PAP, to predict CLD at 24 hours of age.

Several risk factors are associated with the development of CLD. Pulmonary immaturity, reflected by low birthweight and gestational age, is an almost universal finding and provides a setting in which other factors operate. ${ }^{16}$ The damaging effects of oxygen toxicity and barotrauma are generally well accepted as having a central role in pathogenesis, although the nature and importance of antioxidant defences, inflammatory processes, sepsis and fluid overload is less well understood.

Pulmonary artery pressure is frequently increased in infants with RDS and remains persistently high in those who subsequently develop CLD. ${ }^{17}$ Until now it has been unclear 
whether this is an independent association as previous studies have failed to show a consistent association between PAP and markers of disease severity, such as parameters of arterial oxygenation, blood gases, and level of respiratory support. ${ }^{18-20}$ We have shown for the first time that PAP is itself independently associated with CLD in preterm infants.

Although several other studies have attempted to predict CLD using clinical, respiratory, and demographic variables, there are few scoring systems that have been developed and prospectively validated in the era of surfactant and antenatal corticosteroid treatment. ${ }^{3-6}$ Furthermore, we believe that CLD defined as oxygen dependency at 36 weeks of postmenstrual age (rather than at 28 days) is a better predictor of long term respiratory morbidity in extremely preterm infants. ${ }^{15}$ The performance of this score is similar to the CRIB and SNAP scores that have recently been described as indices of initial risk for neonatal mortality. ${ }^{21-22}$ However, with increasing survival of preterm infants, CLD may be an outcome measure which is equally important. In this study deaths which occurred within the neonatal period were included in the CLD outcome group. We believe that this is the most appropriate method of classification as most infants died either primarily from respiratory failure or were receiving considerable respiratory support before death. Nevertheless, when the regression analysis was repeated following exclusion of all deaths, birthweight, $\mathrm{FIO}_{2}$, and AT:RVET(c) remained the only factors independently associated with CLD.

In the development of this score, in addition to an assessment of PAP, we included variables that we had identified as being independent predictors of CLD. Further refinement of this score could have been performed by including other variables which may be associated with CLD and that may have contributed to the overall prediction model. By restricting ourselves to a small number of important and independently predictive variables, we have developed a score that remains simple to calculate without the need for detailed data collection.

Although increased pulmonary blood flow from left to right ductal shunting has been associated with the development of CLD, we were unable to show a significantly higher proportion of symptomatic PDA in infants developing CLD. Our use of prophylactic indomethacin, which became routine during the course of this study, may have influenced the incidence and importance of ductal shunting. ${ }^{23}$ As we were unable to perform detailed echocardiographic assessment of ductal haemodynamics in the development cohort, we cannot comment on the influence of ductal shunting at 24 hours on the subsequent development of CLD. However, there was no association between the presence of ductal shunting and AT:RVET(c) at 24 hours in the validation cohort. This suggests that the association between AT:RVET(c) and the subsequent development of CLD cannot be explained solely on the basis of an increased incidence of ductal shunting.
We have recently published a scoring system to predict CLD on day 4 of life using similar demographic and respiratory parameters. ${ }^{5} \mathrm{Al}-$ though the predictive score performed well, it was only applicable at a time when pulmonary damage was likely to have been established already. We have shown in this study that incorporation of an assessment of PAP at 24 hours of age, in combination with other previously identified factors, permits accurate earlier prediction. Comparison of ROC curves with and without PAP assessment at 24 hours suggests that it is the addition of an assessment of PAP that is responsible for the improved predictive ability, rather than any chance finding through studying a different population of infants at a different time.

This prediction score has been successfully validated prospectively despite the inherent variation present when PAP is assessed using pulmonary flow indices. ${ }^{12}$ We believe that the importance of this variation was minimised by testing the overall performance of the score in a group of infants. It would be inappropriate to use prognostic scores such as this to guide clinical decisions in individuals because of the uncertainty in predicting outcome in any single infant. ${ }^{24}$ This is particularly relevant when using Doppler indices with limited reproducibility such as AT:RVET(c).

During the two year study period, there was also a change in regional referral practices, with a greater proportion of infants born following high risk deliveries being admitted to our unit in the latter part of this study. This may have been responsible for the observed increase in the incidence of CLD in the validation cohort. Additionally, improved early survival (perhaps through the greater use of antenatal corticosteroids) would have allowed the enrolment of some infants into the validation cohort at 24 hours, who would previously have died and therefore have been excluded from the development arm of the study. The consistent performance of this score even when applied prospectively to a slightly different infant population confirms its predictive ability. However, we suggest that further evaluation of this scoring system (ideally in a different neonatal unit with different management policies) is necessary prior to this technique becoming more widely adopted.

This study has shown an independent association between PAP and the development of CLD, but whether this is a causal relation is unknown. Pulmonary hypertension with predominant right to left, extrapulmonary shunting is rare in infants with RDS. ${ }^{25}$ Although this may be an important mechanism of hypoxaemia resulting in increased respiratory support in a few infants, there is at present insufficient evidence to recommend the routine use of pulmonary vasodilator treatment in RDS. It is more likely that pulmonary hypertension is a sensitive marker of pulmonary injury in RDS, rather than being a direct causative factor in the development of CLD.

NVS was supported by the British Heart Foundation (R F Martin Junior Research Fellowship) 
1 Shaw NJ, Gill AB, Weindling AM, Cooke RW. The changing incidence of chronic lung disease. Health Trends incidence of

2 Silverman M. Chronic lung disease of prematurity: are we too cautious with steroids? Eur 7 Pediatr 1994;153:S30-5.

3 Corcoran JD, Patterson CC, Halliday HL, Thomas PS Reduction in the risk of bronchopulmonary dysplasia from 1980-1990: results of a multivariate logistic regression analysis. Eur $\mathcal{F}$ Pediatr 1993; 152:677-81

4 Ryan SW, Wild NJ, Arthur RJ, Shaw BN. Prediction of chronic neonatal lung disease in very low birthweight neonates using clinical and radiological variables. Arch Dis Child 1994;71:F36-9.

5 Ryan SW, Nycyk J, Shaw NJ. Prediction of chronic neonatal lung disease on day 4 of life. Eur F Pediatr 1996;155:66871.

6 Sinkin RA, Cox C, Phelps DL. Prediciting risk for bronchopulmonary dysplaia: selection criteria for clinical bronchopulmonary dysplaia: select

7 Seppanen MP, Kaapa PO, Kero PO. Hemodynamic prediction of complications in neonatal respiratory distress syndrome. $\mathcal{F}$ Pediatr 1995;127:780-5.

8 Gill AB, Weindling AM. Pulmonary artery pressure changes in the very low birthweight infant developing chronic lung disease. Arch Dis Child 1993;68:303-7.

9 Evans NJ, Archer LN. Postnatal circulatory adaptation in healthy term and preterm neonates. Arch Dis Child 1990;65:24-6.

10 Evans NJ, Archer LN. Doppler assessment of pulmonary artery pressure and extrapulmonary shunting in the acute phase of hyaline membrane disease. Arch Dis Child 1991, 66:6-11

11 Akiba T, Yoshikawa M, Otaki S, Kobayashi Y, Nakasato M, Suzuki $\mathrm{H}$ et al.Prediction of peak pulmonary artery pressure by continuous wave Doppler echocardiography in infants and children. Pediatr Cardiol 1988;9:225-9.

12 Subhedar NV, Shaw NJ. Intraobserver variation in Doppler ultrasound assessment of pulmonary artery pressure. Arch Dis Child 1996;75:F59-61.

13 Iyer P, Evans N. Re-evaluation of the left atrial to aortic root ratio as a marker of patent ductus arteriosus. Arch Dis ratio as a marker of patc
Child 1994;70:F112-7.
14 Evans N. Diagnosis of patent ductus arteriosus in the preterm newborn. Arch Dis Child 1993; 68:58-61.

15 Shennan AT, Dunn MS, Ohlsson A, Lennox K, Hoskins EM. Abnormal pulmonary outcomes in premature infants: prediction from oxygen requirement in the neonatal period. Pediatrics 1988; 82:527-32.

16 Cooke RWI. Factors associated with chronic lung disease in preterm infants. Arch Dis Child 1991; 66:776-9.

17 Gill AB, Weindling AM. Raised pulmonary artery pressure in very low birthweight infants requiring supplemental oxygen at 36 weeks after conception. Arch Dis Child 1995;72:F20-2

18 Hamdan $\mathrm{AH}$, Shaw NJ. Changes in pulmonary artery pressure in infants with respiratory distress syndrome following treatment with Exosurf. Arch Dis Child 1995;72:F176 9.

19 Seppanen MP, Kaapa PO, Kero PO, Saraste M. Dopplerderived systolic pulmonary artery pressure in acute neonatal respiratory distress syndrome. Pediatrics 1994;93:76973.

20 Skinner JR, Boys RJ, Hunter S, Hey EN. Pulmonary and systemic arterial pressure in hyaline membrane disease. Arch Dis Child 1991;66:6-11.

21 International Neonatal Network. The CRIB (clinical risk index for babies) score: a tool for assessing initial neonatal risk and comparing performance of neonatal intensive care units. Lancet 1993;342:193-8.

22 Richardson DK, Gray JE, McCormick MC, Workman K, Goldmann DA. Score for neonatal acute physiology: a physiologic severity index for neonatal intensive care. Pediatrics 1993;91:617-23.

23 Fowlie PW. Prophylactic indomethacin: systematic review and meta-analysis. Arch Dis Child 1996; 74:F81-7.

24 Richardson DK, Phibbs CS, Gray JE, McCormick MC, Workman-Daniels K, Goldmann DA. Birthweight and illness severity: independent predictors of neonatal mortality. Pediatrics 1993; 91:969-75.

25 Evans N. Shunts in patients with respiratory distress syndrome. Pediatrics 1993;92:737. 